\title{
Inferior Vena Cava Filter Fractured and Migrated to Left Ventricle
}

\author{
Yuhai Zhang, MD*, ${ }^{1}$ Ming Shen, $M D^{*},{ }^{2}$ Weimin Huang, MD,${ }^{1}$ Liang Wang, $M D,{ }^{1}$ Shixiong Wei, $\mathrm{MD}^{3}$ \\ ${ }^{1}$ Department of Cardiothoracic Surgery, Baotou Clinical Medical College affiliated to Inner Mongolia Medical University, Baotou, \\ Inner Mongolia, China; ${ }^{2}$ Department of Heart Center, The First Hospital of Hebei Medical University, Shijiazhuang, Heibei, China; \\ ${ }^{3}$ Department of Cardiovascular Surgery, Chinese PLA General Hospital, Beijing, China
}

\section{ABSTRACT}

Inferior vena cava (IVC) filters have been widely used to prevent pulmonary emboli in patients with venous thromboembolism. Here we report a rare case of complete pericardial tamponade with severe mitral and tricuspid valve regurgitation due to a fractured IVC filter. A 38-year-old male came to our emergency department with a 19-day history of progressive chest tightness. Chest $\mathrm{x}$-ray revealed a tethering catheter fractured at the level of the junction of the superior vena cava and the right atrium. We performed open surgery and discovered that a steel wire had punctured the atrioventricular septum, the mitral valve, and finally the posterior wall of the left ventricle. The patient recovered quite well and was discharged after 1 week.

\section{INTRODUCTION}

Inferior vena cava (IVC) filters for venous thromboembolism have been used as a means of mechanical intervention for patients with proven pulmonary embolism or with an increased risk of embolization, particularly when anticoagulation is contraindicated [Ollila 2016]. Filter fracture and migration is a known complication associated with IVC filter placement. IVC filters that migrate to the right side of the heart can lodge in the right ventricular wall and progress to penetration, hemopericardium, tamponade, and possible death [Shennib 2014]. Complete pericardial tamponade due to filter strut migration, however, has not been reported. Here we report a rare case of complete pericardial tamponade with severe mitral and tricuspid valve regurgitation due to a fractured IVC filter.

\section{CASE REPORT}

A 38-year-old male came to our emergency department with a 2-week history of progressive chest tightness. Remarkable in his medical history was insertion of an IVC filter via

Received March 30, 2021; accepted April 12, 2021.

*Y. Zhang and M. Shen contributed equally to this article.

Correspondence: Dr. Shixiong Wei, No. 28 Fuxing Road, Haidian Distract, Beijing, 100853, China (e-mail: wei_shixiong@163.com). vena jugularis interna owing to right femoral vein thrombosis 4 months earlier. Unfortunately, the local hospital failed to take out the filter under digital subtraction angiography (DSA) 10 days earlier because of a broken connecting rod.

Pulmonary perfusion scintigraphy revealed pulmonary embolism. After the patient was admitted to intensive care, chest $x$-ray revealed that the tethering catheter had fractured at the level of the junction of the superior vena cava and the right atrium (Figure 1A, red arrow). The upper part of the fractured tethering catheter was removed under DSA, whereas the broken part was shown to be next to the orifice of the inferior vena cava according to ultrasound obtained from the subxiphoid position (Figure 1B). An isolated tiny wire was also detected, piercing through the atrioventricular septum from right atrium to left ventricle into the lateral ventricular wall. Transthoracic echocardiography (TTE) revealed severe mitral and tricuspid regurgitation. A 6-cm steel wire had punctured the atrioventricular septum, the $\mathrm{A} 3$ region of the mitral valve, and finally the posterior wall of the left ventricle (Figure 1C, black arrow).

The left ventricular perforation was repaired with a feltpledgeted 3-0 prolene suture. The prolapse of the mitral valve was repaired with Alfieri end-to-end repair, and annuloplasty was performed with a annuloplasty ring. The tricuspid valve was repaired with an MC3 annuloplasty ring. The filter was removed through an incision in the inferior vena (Figure $1 \mathrm{C}$, red arrow). The patient tolerated the procedure well and was discharged after 1 week. At a 3 -month follow-up appointment, the patient was doing well with no symptoms.

\section{DISCUSSION}

We believe that, after IVC insertion, it is imperative to remove the retrievable IVC filter as soon as protection from pulmonary embolism is no longer needed. It has been reported that the risk/benefit profile favors removal of the filter 29 to 54 days after implant, once the transient risk for pulmonary embolism has passed [Baik 2014; Morales 2013]. The patient's life can be threatened if the IVC filter fractures and migrates to vital organs.

Management of an IVC fragment that migrates into the right ventricle can be challenging. Computed tomography appears to be the best-suited radiologic modality for identifying migrated fragments of an IVC filter within the chest. Correlation between chest pain and the location of migrated struts can assist in making management decisions [Shennib 


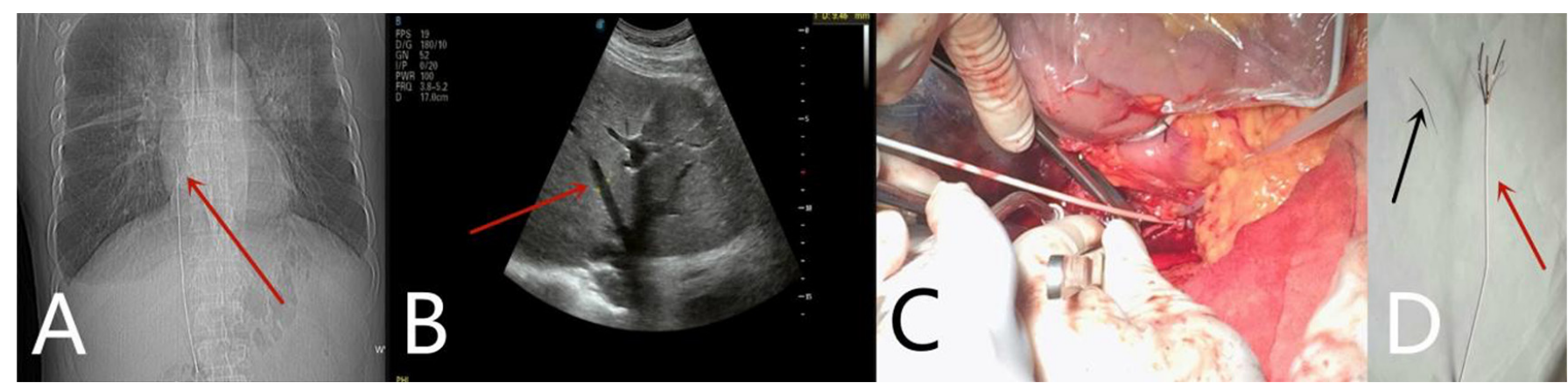

Figure 1. A, X-rays revealed that the IVC filter was fractured at the level of the junction of the superior vena cava and the right atrium. B, TTE revealed that the catheter was visible in the lumen of the posterior segment of IVC near the cardiac entrance. C, The broken IVC filter was removed through an incision in the inferior vena though an abdominal incision. D, The fractured IVC filter and the steel wire.

2014]. Radiologic evaluation of the location and depth of penetration of the strut within the right ventricle may assist in determining whether an endovascular or open surgical approach is more appropriate [Shennib 2014].

Several management strategies have been described pertaining to these migrated filters. A variety of treatment options have been suggested, ranging from conservative pain management to endovascular or open surgical extraction of the migrating struts. However, surgical intervention remains the mainstay of management for migrated IVC filters to the heart [Ollila 2016]. Complete removal of the foreign body is much more readily confirmed surgically, with control of any suspicious bleeding or perforation undertaken in the direct operative field.

\section{REFERENCES}

Baik P, Fourzali R, Salsamendi J, et al. Transventricular migration of an inferior vena cava filter limb. Ann Thorac Surg 2014;97:343.

Morales JP, Li X, Irony TZ, et al. Decision analysis of retrievable inferior vena cava filters in patients without pulmonary embolism. J Vasc Surg Venous Lymphat Disord 2013;1:376-384.

Ollila T, Naeem S, Poppas A, et al. Embolization of inferior vena cava filter tyne and right ventricular perforation: A cardiac missile. Ann Thorac Surg 2016;102:e515-e516.

Shennib H, Bowles B, Hickle K. Migration of a fractured inferior vena cava filter strut to the right ventricle of the heart: A case report. J Cardiothorac Surg 2014;9:183. 